\title{
How Reliable Is the High-Volume Definition in Prostate Cancer Patients: The Potential Game-Changing Role of PSMA
}

\section{Osman Güven ( $\nabla$ osmngvn@gmail.com )}

Giresun University: Giresun Universitesi https://orcid.org/0000-0001-5376-4993

\section{Savaş Karyağar}

Prof Dr Cemil Tascioglu City Hospital: Prof Dr Cemil Tascioglu Sehir Hastanesi

\section{Serdar Arıcı}

Sisli Hamidiye Etfal Training and Research Hospital: Istanbul Sisli Hamidiye Etfal Egitim ve Arastirma Hastanesi

Tamer Ozulker

Prof Dr Cemil Tascioglu City Hospital: Prof Dr Cemil Tascioglu Sehir Hastanesi

Orçun Can

İstinye Üniversitesi: Istinye Universitesi

\section{Research Article}

Keywords: Ga-68 PSMA PET CT, PSMA-TV, prostate cancer, mCSPC, high volume metastatic castration-sensitive prostate cancer

Posted Date: February 17th, 2022

DOI: https://doi.org/10.21203/rs.3.rs-1335464/v1

License: (c) (i) This work is licensed under a Creative Commons Attribution 4.0 International License. Read Full License 


\section{Abstract \\ Purpose}

To evaluate whether metabolic and volumetric data from Ga-68 PSMA PET CT performed during staging of denovo highvolume mCSPC patients who received docetaxel could be used to predict survival.

\section{Methods}

Forty-two denovo high-volume metastatic castration-sensitive prostate cancer patients, who received ADT + Docetaxel and underwent Ga-68 PSMA PET CT for staging, were included in the study. The association between patients' pathological data, all PSA measurements, treatments they received, and the data obtained from Ga-68 PSMA PET CT and progression-free and overall survival were examined. In the univariant analysis performed with the log-rank test, it was evaluated whether the difference between subgroups of variables was statistically significant in terms of survival. Variables found statistically significant were included in the multivariate analysis with the Cox hazard ratio model.

\section{Results}

In the multivariate analysis, PSMA-TV (primary) and PSMA-TV (WB) variables were shown to be independent negative predictors of overall survival. For the threshold value of $19.91 \mathrm{cc}$ obtained for PSMA-TV (primary), HR was calculated as 6.31, $95 \% \mathrm{Cl}: 1.01-39.18, \mathrm{p}: 0.048$. For the threshold value of $1226.5 \mathrm{cc}$ obtained for PSMA-TV(WB) variable, HR was calculated as 58.62, 95\% Cl: 2.55-1344.43, p:0.011. In our study, SUVmax (WB) variable was found to be an independent and negative predictor of progression-free survival. For the determined threshold value of 17.74 , HR was calculated as $16.24,95 \% \mathrm{Cl}$ : $1.18-$ 22.76, p: 0.037.

\section{Conclusion}

Metabolic and volumetric data obtained from Ga-68 PSMA PET CT can be used to predict survival in denovo high-volume mCSPC. Our results show that ADT + Docetaxel receiving patients with high PSMA-TV (WB) values have a significantly worse prognosis. This situation reveals that the high-volume disease definition in the literature is insufficient for this group and that Ga-68 PSMA PET CT can play an important role in demonstrating the heterogeneity within the group.

\section{Introduction}

Approximately $5 \%$ of prostate cancer ( $\mathrm{PCa}$ ) cases, the second most common type of cancer in men, are diagnosed as metastatic[1]. Although it seems like a small percentage, patients with denovo metastatic prostate cancer constitute a very important patient group when their prognostic characteristics and the overall incidence of prostate cancer are taken into account.

Prospective studies on these patients in recent years mainly divide the patient group into two major subgroups as low volume (LV) and high volume (HV)[2-4]. This approach is based on the different prognostic characteristics of subgroups and the different levels of benefit they receive from the treatment approaches. While the combination of androgen deprivation therapy (ADT) with radiotherapy in the LV patient group is the current approach, it is known that Docetaxel (DTX) or second generation antiandrogens provide higher benefits in the HV patient group [2]. The guideline of the European Urology Association (EUA) has also determined their treatment recommendations according to this approach[5].

Although the LV-HV classification based on the extent of the disease rather than the volume provides important benefits, it ignores a critical data by not taking into account the internal heterogeneity of these groups. Since the definition of HV 
disease is based on conventional imaging modalities, there is a lack of studies on the importance of the actual tumor volumes of patients receiving DTX therapy in this group. Gallium-68 Prostate Specific Membrane Antigen - Positron Emission Tomography/Computed Tomography (Ga-68 PSMA PET/CT) has been introduced and preferred as a reliable method in the staging phase of PCa in many centers, making it possible to evaluate metabolic disease volumes in this patient group[6]. PSMA, which is expressed at much higher rates in PCa cells than in normal prostate tissue, is used as a reliable agent in the imaging of prostate cancer[7]. As stated in the EUA guideline, further studies are required to show how the high sensitivity provided by this imaging modality is effective in determining the treatment outcomes of patients[5]. In this study, we aimed both to contribute to the fulfillment of the above-mentioned need and to seek an answer to the question of whether the metabolic and volumetric data obtained from Ga-68 PSMA PET/CT, performed for the purpose of staging, can be used to predict the survival of patients with denovo high-volume metastatic castration-susceptible prostate cancer (mCSPC) receiving DTX treatment.

\section{Materials And Methods}

\section{Patient selection}

The files of patients who underwent Ga-68 PSMA PET/CT for staging of PCa in the Nuclear Medicine Department of our hospital between September 2017 and December 2020 were browsed retrospectively. Out of a total of 862 patient files, 42 patients with no known secondary malignancy, diagnoses confirmed by biopsy, and high-volume denovo metastatic castration-susceptible PCa followed by at least 6 cycles of DTX $(75 \mathrm{mg} / \mathrm{m} 2+$ prednisone $5 \mathrm{mg})$ treatment [5] were included in the study (Figure 1). These patients had also received routine androgen deprivation therapy (ADT). Criteria defined in the ChemoHormonal Therapy Versus Androgen Ablation Randomized Trial for Extensive Disease in Prostate Cancer (CHAARTED) study was used to distinguish between HV and LV while including the patients into the study. Accordingly, the presence of 4 or more bone metastases, at least one of which is outside the vertebral column and pelvis, and/or visceral organ metastases were considered sufficient for the patients to be evaluated as having a high-volume disease[2].

\section{Study protocol}

In this retrospective study, the results of routine diagnostic procedures such as PSA tests, pathology results (ISUP scores), and demographic data of eligible patients who were screened in our clinic were obtained from the hospital's information management system. In addition, the information regarding whether the patients were alive or not and the date of death in case they were dead, was obtained from the Death Notification System (DNS).

As determined by the criteria of the Prostate Cancer Study Group 2 (PCWG-2) for the definition of progression, an increase in three consecutive PSA measurements and a PSA value above $2.0 \mathrm{ng} / \mathrm{ml}$ were adopted. [8] Since there was no progression detected by anatomical imaging without PSA progression in our study group, additional radiological progression criteria were not needed. This study was a graduation/specialization thesis and was carried out with the approval of the Ethics Committee of Prof. Dr. Cemil Taşçıoğlu City Hospital, (protocol number 391).

\section{Ga-68 PSMA PET CT preparation, imaging, and evaluation}

Gallium68 radionuclide was obtained by milking from a Germanium/Gallium (Ge68/Ga68) generator (iThemba LABS@), South Africa) containing $30 \mathrm{mCi}$ (milicurie) of Germanium68. In a fully automated synthesis device (Scintomics@, Germany), $25 \mu \mathrm{g}$ of PSMA I\&T compound was labeled with Ga68 according to the procedure. After the labeling process, the radiochemical purity was evaluated in the liquid chromatography test and the radiopharmaceutical with a result of $95 \%$ and above was considered suitable for application to the patient.

Patients are instructed to drink contrast medium (Omnipaque@ $350 \mathrm{mg} / 50 \mathrm{ml}$ ) diluted with 1.5 liters of water 6 hours before the examination. No additional preparation was required. Ga-68 labeled PSMA compound was administered intravenously at a dose of 1.8-2.2 MBq/kg (0.049-0.060 mCi/ $/ \mathrm{kg})$. 
Patients voided just prior to imaging and they were scanned at the $60^{\text {th }}$ minute with low-dose CT scan for attenuation correction and anatomical correlation, with arms up in the supine position and covering the vertex-upper thigh proximal (including lower extremity if necessary). Immediately afterwards, PET imaging was performed for 3 minutes per bed. All imaging was performed on a Siemens Biograph 6 LSO HI-RES integrated PET/CT device (Siemens Medical Solutions, Chicago, IL, USA). All PET images were acquired in 3D mode and reconstructed with the attenuation-weighted OSEM algorithm (four iterations, eight subgroups). Gaussian smoothing filter ( $5 \mathrm{~mm}$ full width at half-width) was applied to the post-reconstruction images.

Gallium-68 PSMA PET CT images were analyzed as MIP (maximum intensity projection) and cross-sectional images. All areas which visually exceed the normal background activity uptake in these images were evaluated as metastasis when they are outside of the prostate and as primary tumors when in the prostate bed if they do not correspond to the known physiological involvement areas and if the described area does not correspond to any known non-malignant activity involvement (such as inflammation, urinary activity, etc.).

SUVmax, SUVmean, SUVpeak, and PSMA tumor volume (PSMA-TV) (cm3) for each lesion were automatically generated from user-specified VOIs by the Workstation. The borders of the target lesion within the VOI were automatically drawn with isocontour curves and voxels greater than the $45 \%$ threshold of SUVmax in the VOI were defined as the lesion area to measure PSMA-TV and SUVmean[9-10]. In cases where the lesion area observed on CT and the VOI drawn were inconsistent, the threshold value was readjusted to cover the entire lesion and exclude the extralesion tissue. In cases where the borders of PSMA-positive lesions overlapped with the areas of physiological uptake of neighboring organs, the borders of the areas drawn on the VOI were determined manually by using CT images.

The whole body PSMA-TV(WB) value of each patient was defined as the sum of the PSMA-TV of all lesions. Whole-body Total Lesion PSMA, namely TL-PSMA(WB), was obtained by multiplying the SUVmean determined in the volume selected with the isocontour and PSMA-TV(WB). For the primary lesion, the TL-PSMA (primary) value was obtained by multiplying the SUVmean (primary) obtained in the determined VOI area with the PSMA-TV (primary) value. For each patient, SUVmax(primary), SUVmean(primary), SUVpeak(primary), PSMA-TV(primary), TL-PSMA(primary) and SUVmax(WB), SUVmean(WB), SUVpeak(WB), PSMA-TV (WB), and TL-PSMA(WB) data were calculated on ADW 4.7 (GE Healthcare, Chicago, USA) consoles. In addition, bone metastases, visceral metastases, and intra-pelvic-extra-pelvic lymphatic metastases of the patients were also noted while the images were being evaluated.

\section{Statistical method}

SPSS 24.0 for Windows (IBM, New York, USA) package program was used to evaluate the data obtained in the study and the statistical significance limit was determined as $p<0.05$. While descriptive statistics were presented as mean \pm standard deviation for normally distributed numerical variables, categorical variables, ordinal variables, and non-normally distributed numerical variables were presented as median (minimum, maximum).

ROC curves were plotted to determine the appropriate threshold value for continuous variables. This procedure was done separately for overall survival (OS) and progression-free survival (PFS) data for each variable. Threshold values were determined using the Youden index in the drawn ROC curves. After the threshold values of the continuous variables were determined, the survival graphs for the OS and PFS data separately for the variables and the appropriate area under the curve (AUC) in the ROC curves were drawn using the Kaplan-Meier method. At this stage, univariant analyzes were performed using the log-rank method and statistical significance was evaluated. Spearman's correlation analysis was used to evaluate the probability of multicollinearity for the variables with univariate analysis $p<0.25$. The Cox hazard-ratio model was then applied as a multivariate analysis to evaluate the potential independent effects of these variables on OS and PFS.

\section{Results}

\section{Patient characteristics}


The mean age of 42 patients with denovo high volume mCSPC included in the study was 63.52 \pm 9.36 , while basal PSA values were 77.75 (0.37-2314.64) in average. Demographic and clinical characteristics of the patients are shared below. Forty-one of the patients responded to the 6 cycles of DTX treatment and progression was observed in only 1 patient despite the treatment. The median follow-up period in our study group was 759 (196-1771) days (Table 1).

According to the data obtained from Kaplan-Meier graphs drawn to calculate the overall OS and PFS times of the study group, the median PFS was 296 days and the $95 \% \mathrm{Cl}$ was calculated as $149-442$ days. The mean OS was calculated as $1217 \pm 117$ days in the study group, which did not reach the median duration in OS. In our study group, 1-year PFS was $46.1 \pm 7.9 \%$, while 4 -year PFS was calculated as $20.5 \% \pm 8.4 \%$. The 1 -year OS was $92.5 \pm 4.2 \%$, while the 4 -year OS was $52.4 \pm 10.5 \%$.

\section{Ga-68 PSMA PET CT findings}

When the Ga-68 PSMA PET CT images of the patients in the study group were examined, 38 (90.47\%) lymphatic, 36 (85.71\%) skeletal system, and 9 (21.42\%) visceral organ metastases were detected.

A total of 10 different semi-quantitative parameters were calculated for each patient, 5 of which reflect the primary lesion and 5 reflect the whole body data on Ga-68 PSMA PET CT images. The median (minimum-maximum) values of the metabolic semi-quantitative data obtained throughout the study group are presented in the table (Table 2).

\section{Univariate analysis}

A statistically significant difference was found in terms of PFS in the groups above and below the determined threshold values for Basal PSA, nadir PSA, $2^{\text {nd }}$ generation antiandrogen use, PSMA-TV(primary), TL-PSMA(primary), SUVmax(WB), PSMA-TV(WB), and TL-PSMA(WB) variables. In the analyses for OS, there was a statistically significant difference between the groups above and below the threshold values determined for nadir PSA, PSMA-TV (primary), TL-PSMA (primary), PSMATV (WB), TL-PSMA (WB), and ECOG scores. The threshold values used in these analyses, their sensitivities, specificities, and all $\mathrm{p}$ values obtained are presented in Table 3 .

\section{Multivariate analysis}

In the correlation analysis, no more than $60 \%$ correlation was found between the variables and it was accepted that there was no multicollinearity problem. Thereupon, Cox hazard ratio regression analysis was performed for PFS and OS in separate groups.

Among the variables included in the model studied for PFS, only the SUVmax(WB) variable was found to be statistically significant (p:0.037). The Hazard Ratio (HR) calculated for the SUVmax(WB) variable was 16.24 and the $95 \%$ confidence interval (Cl) was found to be 1.18-22.76. (Figure 2) According to this data obtained, the probability of progression in the group with SUVmax (WB) $>17.74$ during the follow-up period in the study group was calculated as 16.24 times that of the group with SUVmax(WB) $<17.74$ (Table 4).

Among the variables included in the studied model for overall survival, only PSMA-TV(primary) and PSMA-TV(WB) variables were found to be statistically significant ( $p$ values 0.048 and 0.011 , respectively). Hazard Ratio (HR), calculated for PSMA-TV (primary), which is one of the statistically significant variables, was found to be 6.31 and $95 \%$ confidence interval (Cl) was 1.01-39.18. (Figure 3) According to this data, the probability of death in the group with PSMA-TV (primary) $>19.91$ cc during the follow-up period in the study group was found to be 6.31 times that of the group with PSMA-TV (primary) $<19.91$. Hazard Ratio (HR), calculated for PSMA-TV(WB), which was another significant variable, was found as 58.62 and $95 \%$ confidence interval (Cl) was 2.55-1344.43. (Figure 4) According to this data obtained, the probability of death in the group with PSMATV(WB) $>1226.5$ cc during the follow-up period in the study group was 58.26 times higher than in the group with PSMATV(WB) <1226.5 (Table 5). 
Among the variables evaluated in the study group, PSMA-TV(primary) and PSMA-TV(WB) variables stand out as independent negative predictors for OS and SUVmax(WB) variable for PFS. The survival times and $p$ values calculated for the threshold values for these variables are presented in the table (Table 6).

\section{Discussion}

Since the majority of prostate cancer patients are not metastatic at the time of the first diagnosis, the treatment approach includes definitive treatments such as radiotherapy or surgery. However, the patient group that is metastatic at the time of diagnosis or later requires a different approach in terms of treatment. The only data in the literature on OS obtained under ADT + DTX in the denovo high-volume mCSPC group, on which was focused in our study, is presented in the article in which the long-term results of the prospective CHAARTED study were published. Based on the data in this article, OS achieved in the denovo high-volume mCSPC group is 48 months [11]. The median OS of 1217 days (40.56 months) obtained in our study group is significantly lower than that was obtained in the literature. In our study, the median PSMA-TV(WB) value was found to be $243.2 \mathrm{cc}$ and the difference between the literature and our study group is thought to be due to this high tumor burden.

Almost all of the studies in the literature on hormone-sensitive metastatic prostate cancer patients focus on the efficacy of combined therapies and the different profiles regarding the benefit from the treatment of low-volume and high-volume patient groups $[2-3,12]$. However, another important issue here is the heterogeneity of these groups within themselves. Our current study differs from the literature in that it evaluates the high-volume group within itself and draws attention to this heterogeneity. The definition of high volume is not sufficient on its own when OS is regarded.

In our study, the HR calculated in terms of overall survival between the groups above and below the threshold value of 1226.5 cc obtained in PSMA-TV (WB) value was found to be 58.62 and $95 \% \mathrm{Cl}$ was $2.55-1344.43$. This finding indicates that, within the broadly described high-volume disease group, a subgroup (group with a total tumor volume in the body $>1226.5 \mathrm{cc}$ ) can be differentiated, with a worse prognosis than the general group despite standard treatment. Studies in the nuclear medicine literature generally focus on treatment response and the number of studies investigating OS and PFS are limited. In a study examining the response to DTX treatment and survival data in metastatic castration-resistant prostate cancer (mCRPC) patients, it was found that high TV-PSMA (p: 0.024), high age (p: 0.016), and high LDH ( $<<0.001)$ values were negative predictors for OS [13]. This study differs from ours by involving patients with castration-resistant PCa and not focusing on the distinction between low and high volume. The TV-PSMA variable (corresponding to the PSMA-TV(WB) variable in our study), which was found to be significant in terms of time to progression, was not found to be significant in terms of PFS in our study. In another study in the literature, Zou et al. found TL-PSMA(WB) and Gleason score as predictive variables for PFS [14]. However, when the composition of the study group is examined, it is seen that 29 of the 59 patients showed involvement only in the prostate. The difference between the patient groups in these cited studies and our study makes it difficult to compare the results obtained individually and it is understood that prospective studies with larger series are needed in this area.

Although there is a general consensus on the prognostic value of whole body tumor volume, there is an ongoing debate on the predictive value of primary tumor volume. Studies have shown that primary tumor volume has no effect on survival in groups with low Gleason scores or does not show any correlation with survival data [15-16]. However, it has been demonstrated by studies on pathology preparations that, as the Gleason score of the disease increases, there appears a significant relationship between survival and tumor volume, especially in moderate and high-risk local diseases. [16-17] For instance, in a study by Knoedler et al., 13687 patients were monitored for an average of 9.4 years and it was shown that primary tumor volume was associated with the risk of death (HR 1.29; $P<0.0001)$ [18]. One of the crucial points here is how the tumor volume is measured. While the majority of studies in the literature reporting that primary tumor volume is not a predictor of survival data are based on the visual evaluation included in pathology reports, [19] it is noticed that computerbased methods are generally used in studies that indicate a statistically significant relationship $[16,18]$. Therefore, it is necessary to be cautious about the studies performed with methods such as visual evaluation, which do not have sufficient accuracy in the evaluation of primary tumor volume. The problems encountered in the previous literature are not limiting to 
our study because we used computer algorithms based on the focus with the highest PSMA uptake and calculating the PSMA uptake areas determined over this as independent voxels.

It has long been known that there is an inverse relationship between tumor burden and survival of patients. The PSMATV(WB) parameter, which is used to calculate the whole body tumor burden thanks to Ga-68 PSMA PET CT, provided results that support and make usable our basic knowledge of tumor biology in this respect. Especially in staging patients who have not received treatment before, when homogenization is provided in terms of treatment, it is seen that the whole body tumor burden at baseline is an independent predictor of OS.

One of the striking findings in our study is that TL-PSMA was not found to be statistically significant, while the concomitant PSMA-TV value was significant. As can be seen in the literature, although not in terms of survival, it is known that these variables correlate in clinical situations such as progression detection and treatment response failure [9-10, 20-22]. In our study group, mild to moderate PSMA involvement was detected especially in patients with extensive bone metastases and we conclude that this might have affected the correlation between PSMA-TV and TL-PSMA values in the study.

When the median follow-up time of our study and the inaccessibility of median survival especially in low-volume groups are taken into account, it can be deduced that evaluations with longer follow-up results will be beneficial. Another one of our serious limitations is that our study group did not include many patients with the HV disease and a low tumor burden in volumetric calculations. Series with a more balanced volumetric distribution, including this subgroup, can provide more detailed data. The retrospective nature of our study was also one of our most important limitations. It is clear that this study, which was conducted with 42 patients, needs to be supported by prospective studies with larger series.

\section{Conclusion}

In our study, in which metabolic data obtained from Gallium-68 PSMA PET CT studies were examined, it was found that SUVmax (WB) in terms of PFS and PSMA-TV (primary) and PSMA-TV(WB) variables in terms of OS had negative predictive values. Our study shows that Denovo high-volume MCSPC patients are a heterogeneous group within themselves and this indicates that this group can be further divided into subcategories on the basis of metabolic volumes.

In this context, low-high volume disease definitions based on assessment of the extent of the disease in the body with conventional methods are insufficient when classifying the patient group. Ga-68 PSMA PET CT stands out as an important tool that can fill the gap in this area.

\section{Declaration}

\section{Funding}

The authors declare that no funds, grants, or other support were received during the preparation of this manuscript.

\section{Competing Interests}

The authors have no relevant financial or non-financial interests to disclose.

\section{Author Contributions}

All authors contributed to the study conception and design. Material preparation, data collection and analysis were performed by Osman Güven. The first draft of the manuscript was written by Osman Güven and all authors commented on previous versions of the manuscript. All authors read and approved the final manuscript.

\section{Data Availability}


The datasets generated during and/or analysed during the current study are available from the corresponding author on reasonable request.

\section{Ethics approval}

This study was performed in line with the principles of the Declaration of Helsinki. Approval was granted by the Ethics Committee of Prof. Dr. Cemil Taşçıoğlu City Hospital (03.05.2021/391).

\section{Consent to participate}

Informed consent was obtained from all individual participants included in the study.

\section{Reference}

1. Bernard B, Burnett C, Sweeney CJ, Rider JR, Sridhar SS. Impact of Age at Diagnosis of De novo Metastatic Prostate Cancer on Survival. Cancer. 2020;126(5):986-993. doi:10.1002/cncr.32630

2. Sweeney CJ, Chen YH, Carducci M, Liu G, Jarrard DF, Eisenberger M, et al. Chemohormonal Therapy in Metastatic Hormone-Sensitive Prostate Cancer. N Engl J Med. 2015;373(8):737-746. doi:10.1056/NEJMoa1503747

3. Vale CL, Burdett S, Rydzewska LHM, Albiges L, Clarke NW, Fisher D, et al. Addition of Docetaxel or Bisphosphonates to Standard of Care in Men With Localised or Metastatic, Hormone-Sensitive Prostate Cancer: A Systematic Review And Meta-Analyses of Aggregate Data. Lancet Oncol. 2016;17(2):243-256. doi:10.1016/S1470-2045(15)00489-1

4. Clarke NW, Ali A, Ingleby FC, Holye A, Amos CL, Attard G, et al. Addition of Docetaxel to Hormonal Therapy in Low- And High-Burden Metastatic Hormone Sensitive Prostate Cancer: Long-Term Survival Results From the STAMPEDE Trial. Ann Oncol. 2019;30(12):1992-2003. doi:10.1093/annonc/mdz396

5. Mottet N, van den Bergh RCN, Briers E, van den Broeck T, Cumberbatch MG, Santis MD, et al. EAU-EANM-ESTRO-ESURSIOG Guidelines on Prostate Cancer-2020 Update. Part 1: Screening, Diagnosis, and Local Treatment with Curative Intent. Eur Urol. 2021;79(2):243-262. doi:10.1016/j.eururo.2020.09.042

6. Schmuck S, von Klot CA, Henkenberens C, Sohns JM, Christiansen H, Wester HJ, et al. Initial Experience with Volumetric 68Ga-PSMA I\&T PET/CT for Assessment of Whole-Body Tumor Burden as a Quantitative Imaging Biomarker in Patients with Prostate Cancer. J Nucl Med. 2017;58(12):1962-1968. doi:10.2967/jnumed.117.193581

7. Sweat SD, Pacelli A, Murphy GP, Bostwick DG. Prostate-Specific Membrane Antigen Expression is Greatest in Prostate Adenocarcinoma and Lymph Node Metastases. Urology. 1998;52(4):637-640. doi:10.1016/s0090-4295(98)00278-7

8. Scher HI, Halabi S, Tannock I, Morris M, Sternberg CN, Carducci MA, et al. Design and End Points of Clinical Trials for Patients With Progressive Prostate Cancer and Castrate Levels of Testosterone: Recommendations of the Prostate Cancer Clinical Trials Working Group. J Clin Oncol. 2008;26(7):1148-1159. doi:10.1200/JC0.2007.12.4487

9. Schmuck S, von Klot CA, Henkenberens C, Sohns JM, Christiansen H, Wester HJ, et al. Initial Experience with Volumetric 68Ga-PSMA I\&T PET/CT for Assessment of Whole-Body Tumor Burden as a Quantitative Imaging Biomarker in Patients with Prostate Cancer. J Nucl Med. 2017;58(12):1962-1968. doi:10.2967/jnumed.117.193581

10. Schmidkonz C, Cordes M, Schmidt D, Bäuerle T, Goetz TI, Beck M, et al. 68Ga-PSMA-11 PET/CT-Derived Metabolic Parameters for Determination of Whole-Body Tumor Burden and Treatment Response in Prostate Cancer. Eur J Nucl Med Mol Imaging. 2018;45(11):1862-1872. doi:10.1007/s00259-018-4042-z

11. Kyriakopoulos CE, Chen YH, Carducci MA, Liu G, Jarrard DF, Hahn NM, et al. Chemohormonal Therapy in Metastatic Hormone-Sensitive Prostate Cancer: Long-Term Survival Analysis of the Randomized Phase III E3805 CHAARTED Trial. J Clin Oncol. 2018;36(11):1080-1087. doi:10.1200/JC0.2017.75.3657 
12. Gravis G, Fizazi K, Joly F, Priou F, Esterni B, Latorzeff I, et al. Androgen-Deprivation Therapy Alone or With Docetaxel in Non-Castrate Metastatic Prostate Cancer (GETUG-AFU 15): A Randomised, Open-Label, Phase 3 Trial. Lancet Oncol. 2013;14(2):149-158. doi:10.1016/S1470-2045(12)70560-0

13. Has Simsek D, Kuyumcu S, Karadogan S, Oflas M, Isik EG, Ozkan ZG, et al. Can PSMA-Based Tumor Burden Predict Response to Docetaxel Treatment in Metastatic Castration-Resistant Prostate Cancer?. Ann Nucl Med. 2021;35(6):680690. doi:10.1007/s12149-021-01610-x

14. Zou Q, Jiao J, Zou MH, Li MZ, Yang T, Xu L, et al. Semi-Automatic Evaluation of Baseline Whole-Body Tumor Burden as an Imaging Biomarker of 68Ga-PSMA-11 PET/CT in Newly Diagnosed Prostate Cancer. Abdom Radiol (NY).

2020;45(12):4202-4213. doi:10.1007/s00261-020-02745-7

15. Merrill MM, Lane BR, Reuther AM, Zhou M, Magi-Galluzzi C, Klein EA. Tumor Volume Does Not Predict for Biochemical Recurrence After Radical Prostatectomy in Patients With Surgical Gleason Score 6 or Less Prostate Cancer. Urology. 2007;70(2):294-298. doi:10.1016/j.urology.2007.03.062

16. Brooks JD, Tibshirani R, Ferrari M, Presti Jr. JC, Gill H, King CR. The Impact of Tumor Volume on Outcomes after Radical Prostatectomy: Implications for Prostate Cancer Screening, Open Prost. Cancer J.,2014;1(1):1-8.

17. Chung BI, Tarin TV, Ferrari M, Brooks JD. Comparison of Prostate Cancer Tumor Volume and Percent Cancer in Prediction of Biochemical Recurrence and Cancer Specific Survival. Urol Oncol. 2011;29(3):314-318. doi:10.1016/j.urolonc.2009.06.017

18. Knoedler JJ, Karnes RJ, Thompson RH, Rangel LJ, Bergstralh EJ, Boorjian SA. The Association of Tumor Volume With Mortality Following Radical Prostatectomy. Prostate Cancer Prostatic Dis. 2014;17(2):144-148. doi:10.1038/pcan.2013.61

19. Porten SP, Cooperberg MR, Carroll PR. The Independent Value of Tumour Volume in a Contemporary Cohort of Men Treated With Radical Prostatectomy for Clinically Localized Disease. BJU Int. 2010;105(4):472-475. doi:10.1111/j.1464410X.2009.08774.x

20. Acar E, Özdoğan Ö, Aksu A, Derebek E, Bekiş R, Çapa Kaya G. The Use of Molecular Volumetric Parameters For the Evaluation of Lu-177 PSMA I\&T Therapy Response and Survival. Ann Nucl Med. 2019;33(9):681-688. doi:10.1007/s12149-019-01376-3

21. Liu C, Liu T, Zhang N, Liu Y, Li N Du P, et al. 68Ga-PSMA-617 PET/CT: A Promising New Technique for Predicting Risk Stratification and Metastatic Risk of Prostate Cancer Patients. Eur J Nucl Med Mol Imaging. 2018;45(11):1852-1861. doi:10.1007/s00259-018-4037-9.

22. Karyagar SS, Karyagar S, Guven O. Correlations of the 68Ga-PSMA PET/CT Derived Primary Prostate Tumor PSMA Expression Parameters and Metastatic Patterns in Patients with Gleason Score $>7$ Prostate Cancer. Hell J Nucl Med. 2020;23(2):120-124. doi:10.1967/s002449912100

\section{Tables}

Table 1: Demographic and Clinical Characteristics of the Patients 


\begin{tabular}{|c|c|}
\hline \multicolumn{2}{|l|}{ Demographic and Clinical Characteristics of the Patients } \\
\hline Age Mean $\pm S D$ & $63,52 \pm 9,36$ \\
\hline Pre-DTX PSA Level (ng/ml) Median (range) & $77,75(0,37-2314,64)$ \\
\hline Nadir PSA Value After DTX (ng/ml) Median (range) & $0,76(0,01-808,79)$ \\
\hline Time Interval Between Biopsy and PET-CT (gün) Mean \pm SD & $20,12 \pm 8,45$ \\
\hline Time Interval Between PET BT and DTX Treatment (days) Mean \pm SD & $16,82 \pm 6,46$ \\
\hline \multicolumn{2}{|l|}{ ECOG } \\
\hline 0 & $15(\% 35,71)$ \\
\hline 1 & $27(\% 64,29)$ \\
\hline 2 & $0(\% 0)$ \\
\hline \multicolumn{2}{|l|}{ ISUP } \\
\hline 3 & $3(\% 7,14)$ \\
\hline 4 & $13(\% 30,95)$ \\
\hline 5 & $26(\% 61,9)$ \\
\hline \multicolumn{2}{|l|}{ Other Treatments } \\
\hline Palliative RT Before DTX n(\%) & $14(\% 33,3)$ \\
\hline Palliative RT After DTX n(\%) & $16(\% 38,09)$ \\
\hline Second Generation Anti-androgens After DTX n(\%) & $15(35,71)$ \\
\hline Progression n(\%) & $28(\% 66,6)$ \\
\hline Death n(\%) & $13(30,95)$ \\
\hline
\end{tabular}

Table 2: PET-BT Based Variables (Median, Maximum, Minimum)

\begin{tabular}{|llll|}
\hline & Median & Maximum & Minimum \\
\hline SUVmax(primary) & 14,08 & 77,34 & 3,39 \\
\hline SUVmean(primary) & 6,71 & 26,53 & 1,76 \\
\hline SUVpeak(primary) & 10,52 & 55,95 & 2,96 \\
\hline PSMA - TV(primary) & $16,35 \mathrm{cc}$ & 421,00 & 5,33 \\
\hline TL-PSMA(primary) & 136,95 & 1371,31 & 10,28 \\
\hline SUVmax(WB) & 29,26 & 113,53 & 4,33 \\
\hline SUVmean(WB) & 7,01 & 20,66 & 1,46 \\
\hline SUVpeak(WB) & 20,30 & 88,91 & 5,19 \\
\hline PSMA-TV(WB) & $243,20 \mathrm{cc}$ & 2967,00 & 41,74 \\
\hline TL-PSMA(WB) & 1356,65 & 26020,59 & 131,54 \\
\hline
\end{tabular}


Table 3: Results of univariant analysis

\begin{tabular}{|c|c|c|c|c|c|c|c|c|}
\hline & \multicolumn{4}{|l|}{ PFS } & \multicolumn{4}{|l|}{ os } \\
\hline & Sensitivity & Specificity & $\begin{array}{l}\text { Threshold } \\
\text { Value }\end{array}$ & p & Sensitivity & Specificity & $\begin{array}{l}\text { Threshold } \\
\text { Value }\end{array}$ & $p$ \\
\hline Age (Years) & $78.6 \%$ & $28.6 \%$ & 58,5 & 0,803 & $61.5 \%$ & $72.4 \%$ & 65.5 & 0,228 \\
\hline Basal PSA (ng/ml) & $85.7 \%$ & $50 \%$ & 24,56 & $0,001 *$ & $84.6 \%$ & $31.2 \%$ & 24.56 & 0,096 \\
\hline Nadir PSA (ng/ml) & $82.1 \%$ & $64.3 \%$ & 0,26 & $0,000 *$ & $69.2 \%$ & $69.1 \%$ & 1 & $0,038 *$ \\
\hline Pre-Treatment RT & $\star \star$ & $\star \star$ & Yes/No & 0,54 & ** & ** & Yes/No & 0,350 \\
\hline Post-Treatment RT & ** & ** & Yes/No & 0,32 & ** & ** & Yes/No & 0,836 \\
\hline $\begin{array}{l}\text { Second-generation } \\
\text { Anti-androgens }\end{array}$ & ** & ** & Yes/No & $0,012^{*}$ & ** & ** & Yes/No & 0,786 \\
\hline SUVmax(primary) & $67.9 \%$ & $05 \%$ & 10,52 & 0,817 & $100.0 \%$ & $8.97 \%$ & 5,42 & 0,454 \\
\hline SUVmean(primary) & $89.3 \%$ & $35.7 \%$ & 3,64 & 0,352 & $84.5 \%$ & $24.1 \%$ & 3,84 & 0,743 \\
\hline SUVpeak(primary) & $78.6 \%$ & $42.9 \%$ & 7,17 & 0,751 & $100.0 \%$ & $9.6 \%$ & 3,55 & 0,633 \\
\hline $\begin{array}{l}\text { PSMA-TV(primary) } \\
\text { (cc) }\end{array}$ & $57.1 \%$ & $92.9 \%$ & 18,83 & $0,012^{*}$ & $76.9 \%$ & $79.3 \%$ & 19,91 & $0,006^{*}$ \\
\hline TL-PSMA(primary) & $82.1 \%$ & $78.6 \%$ & 91,75 & $0,005^{*}$ & $92.3 \%$ & $51.7 \%$ & 91,75 & $0,007 *$ \\
\hline SUVmax(WB) & $89.3 \%$ & $50 \%$ & 17,74 & $0,019 *$ & $84.5 \%$ & $34.5 \%$ & 20,21 & 0,413 \\
\hline SUVmean(WB) & $64.3 \%$ & $64.3 \%$ & 6,75 & 0,274 & $61.5 \%$ & $48.3 \%$ & 6,75 & 0,921 \\
\hline SUVpeak(WB) & $82.1 \%$ & $50 \%$ & 12,07 & 0,112 & $76.9 \%$ & $37.9 \%$ & 13,43 & 0,452 \\
\hline PSMA-TV(WB) (cc) & $75 \%$ & $71.4 \%$ & 169,35 & $0,001 *$ & $53.8 \%$ & $89.7 \%$ & 1226.5 & 0,006 * \\
\hline TL-PSMA(WB) & $71.4 \%$ & $85.7 \%$ & 1173,61 & $0,000 *$ & $38,50 \%$ & $93.1 \%$ & 10828.5 & $0,016 *$ \\
\hline $\begin{array}{l}\text { Intrapelvic } \\
\text { Lymphatic } \\
\text { Metastasis }\end{array}$ & $\star \star$ & ** & Var/Yok & 0,639 & $\star \star$ & ** & Var/Yok & 0,858 \\
\hline $\begin{array}{l}\text { Extrapelvic } \\
\text { Lymphatic } \\
\text { Metastasis }\end{array}$ & $\star \star$ & $\star \star$ & Var/Yok & 0,148 & $\star \star$ & ** & Var/Yok & 0,640 \\
\hline Bone Metastasis & ** & $\star \star$ & Var/Yok & 0,051 & $\star \star$ & ** & Var/Yok & 0,235 \\
\hline Visceral Metastaz & ** & ** & Var/Yok & 0,483 & ** & ** & Var/Yok & 0,291 \\
\hline ECOG & ** & ** & $0 / 1$ & 0,185 & ** & ** & $0 / 1$ & $0,006 *$ \\
\hline $\begin{array}{l}\text { Progression Time } \\
\text { (days) }\end{array}$ & & & & & $100.0 \%$ & $3.4 \%$ & 122,5 & 0,364 \\
\hline
\end{tabular}

${ }^{*} \mathrm{p}<0,005$

** Nominal Variables 
Table:4 Multivariant Analysis (PFS)

\begin{tabular}{|lclll|}
\hline & p value & HR & \multicolumn{2}{c|}{$95,0 \%$ Cl for HR } \\
\cline { 5 - 6 } & & & Lower Bound & Upper Bound \\
\hline Basal PSA &, 244 & 2,604 &, 520 & 13,035 \\
\hline ECOG &, 355 &, 606 &, 210 & 1,753 \\
\hline Nadir PSA &, 120 & 3,905 &, 701 & 21,769 \\
\hline Second-generation Anti-androgens &, 268 &, 570 &, 211 & 1,542 \\
\hline PSMA-TV(primary) &, 105 & 2,695 &, 812 & 8,946 \\
\hline TL-PSMA(primary) &, 597 &, 639 &, 121 & 3,364 \\
\hline SUVmax(WB) &, $037 *$ & 16,241 & $\mathbf{1 , 1 8 4}$ & $\mathbf{2 2 2 , 7 6 7}$ \\
\hline SUVpeak(WB) &, 055 &, 102 &, 010 & 1,053 \\
\hline PSMA-TV(WB) &, 777 &, 747 &, 100 & 5,607 \\
\hline TL-PSMA(WB) &, 192 & 2,847 &, 591 & 13,723 \\
\hline Extrapelvic Lymphatic Metastasis &, 148 & 2,342 &, 740 & 7,413 \\
\hline Bone Metastasis &, 145 & 6,390 &, 527 & 77,505 \\
\hline
\end{tabular}

Table:5 Multivariant Analysis (OS)

\begin{tabular}{|c|c|c|c|c|}
\hline & \multirow[t]{2}{*}{ p değeri } & \multirow[t]{2}{*}{ HR } & \multicolumn{2}{|c|}{$95,0 \% \mathrm{Cl}$ for $\mathrm{HR}$} \\
\hline & & & Lower Bound & Upper Bound \\
\hline Age & , 162 & 1,084 & ,968 & 1,213 \\
\hline Basal PSA & ,427 & 2,313 & 292 & 18,339 \\
\hline Nadir PSA & ,218 & ,286 & ,039 & 2,093 \\
\hline PSMA-TV(primary) &, $048 *$ & 6,318 & 1,019 & 39,187 \\
\hline TL-PSMA(primary) & ,767 & 1,427 & ,136 & 15,001 \\
\hline PSMA-TV(WB) &, $011^{*}$ & 58,629 & 2,557 & 1344,439 \\
\hline TL-PSMA(WB) & ,869 & 1,237 & 099 & 15,500 \\
\hline Bone Metastasis & ,984 & 3785,491 & ,000 & N/A \\
\hline ECOG & ,936 & 1519397,2 &, 000 & $\mathrm{~N} / \mathrm{A}$ \\
\hline
\end{tabular}

Table 6: Variables that were significant at the end of the multivariate analysis and the survival times obtained 


\begin{tabular}{|c|c|c|c|}
\hline & Threshold & os & p \\
\hline \multirow[t]{2}{*}{ PSMA-TV(primary) } & $>19,91 \mathrm{cc}$ & 759 (641-876) days & \multirow[t]{2}{*}{0,048} \\
\hline & $<19,91 \mathrm{cc}$ & $1299,55 \pm 95,25^{\star}$ days & \\
\hline \multirow[t]{3}{*}{ PSMA-TV(WB) } & $>1226,5 \mathrm{cc}$ & 519 (61-398) days & \multirow[t]{2}{*}{0,011} \\
\hline & $<1226,5 \mathrm{cc}$ & $1364,34 \pm 135,91^{*}$ days & \\
\hline & Threshold & PFS & $p$ \\
\hline \multirow[t]{2}{*}{ SUVmax(WB) } & $>17,74$ & 263 (22-218) days & \multirow[t]{2}{*}{0,037} \\
\hline & $<17,74$ & $490 \pm 49,15^{\star}$ dasy & \\
\hline
\end{tabular}

* Survival statistics are given as mean \pm SD instead of the median (range) in subgroups whose median survival time could not be reached.

\section{Figures}

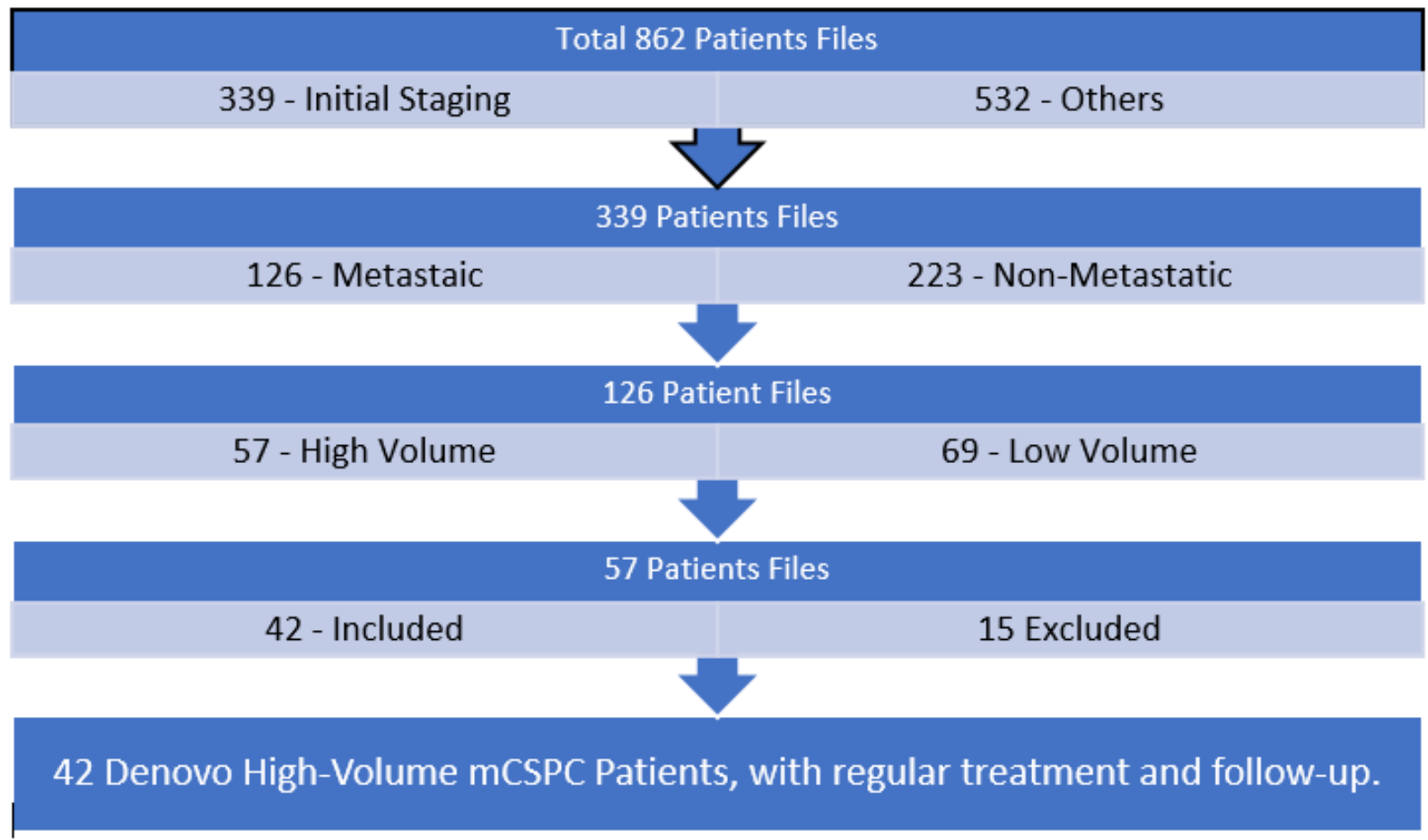

\section{Figure 1}

Legend not included with this version 


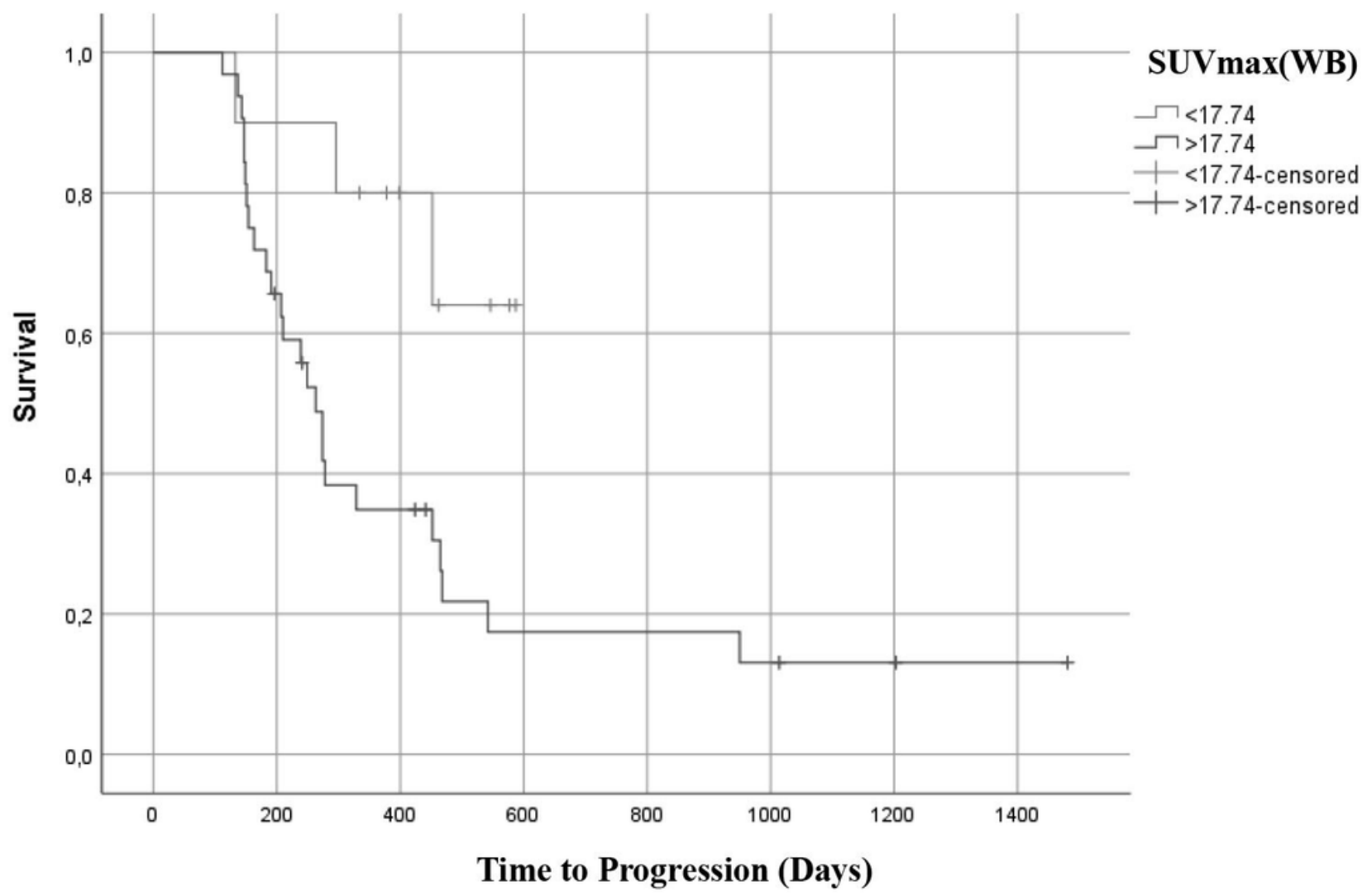

Figure 2

Legend not included with this version

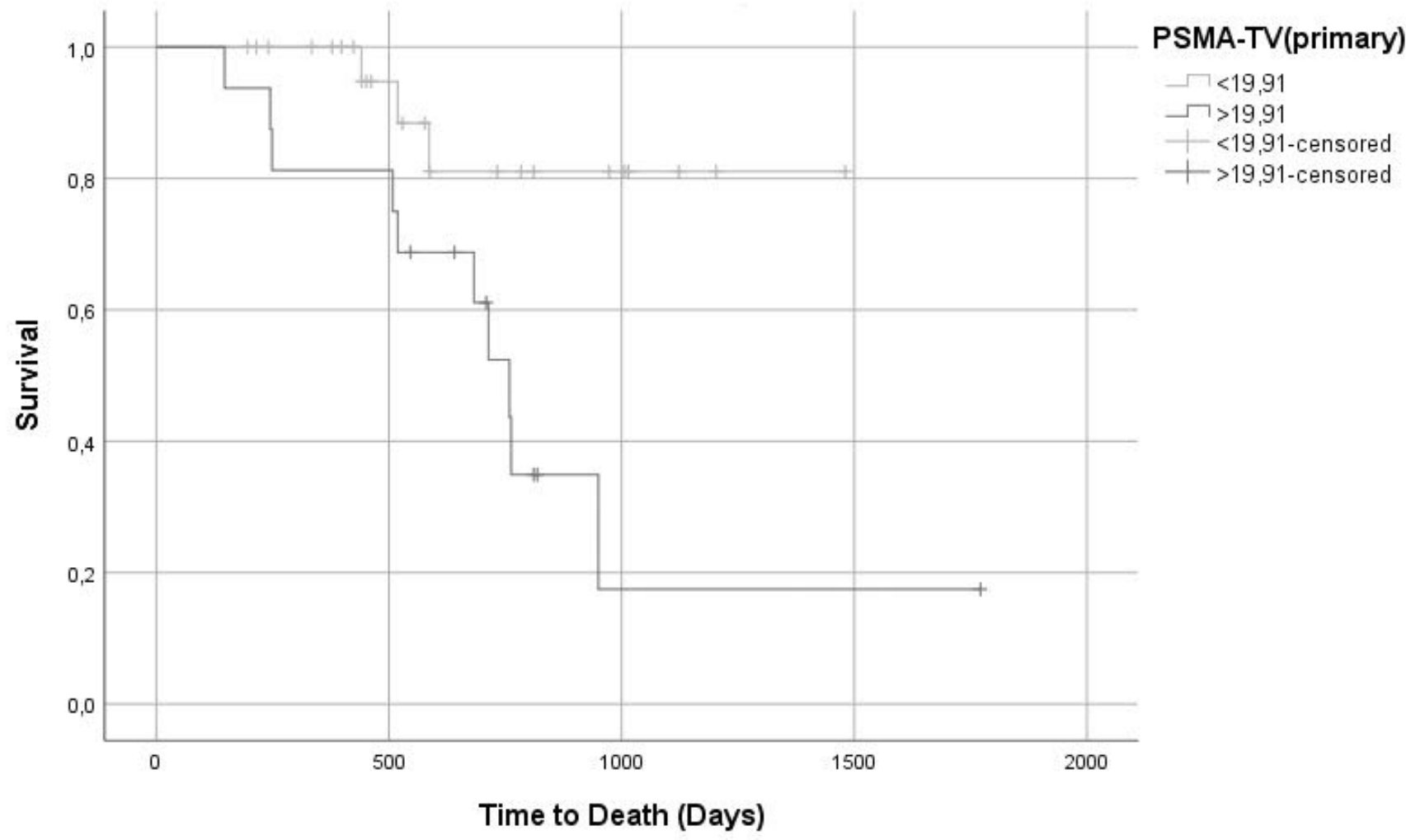

Page 14/15 


\section{Figure 3}

Legend not included with this version

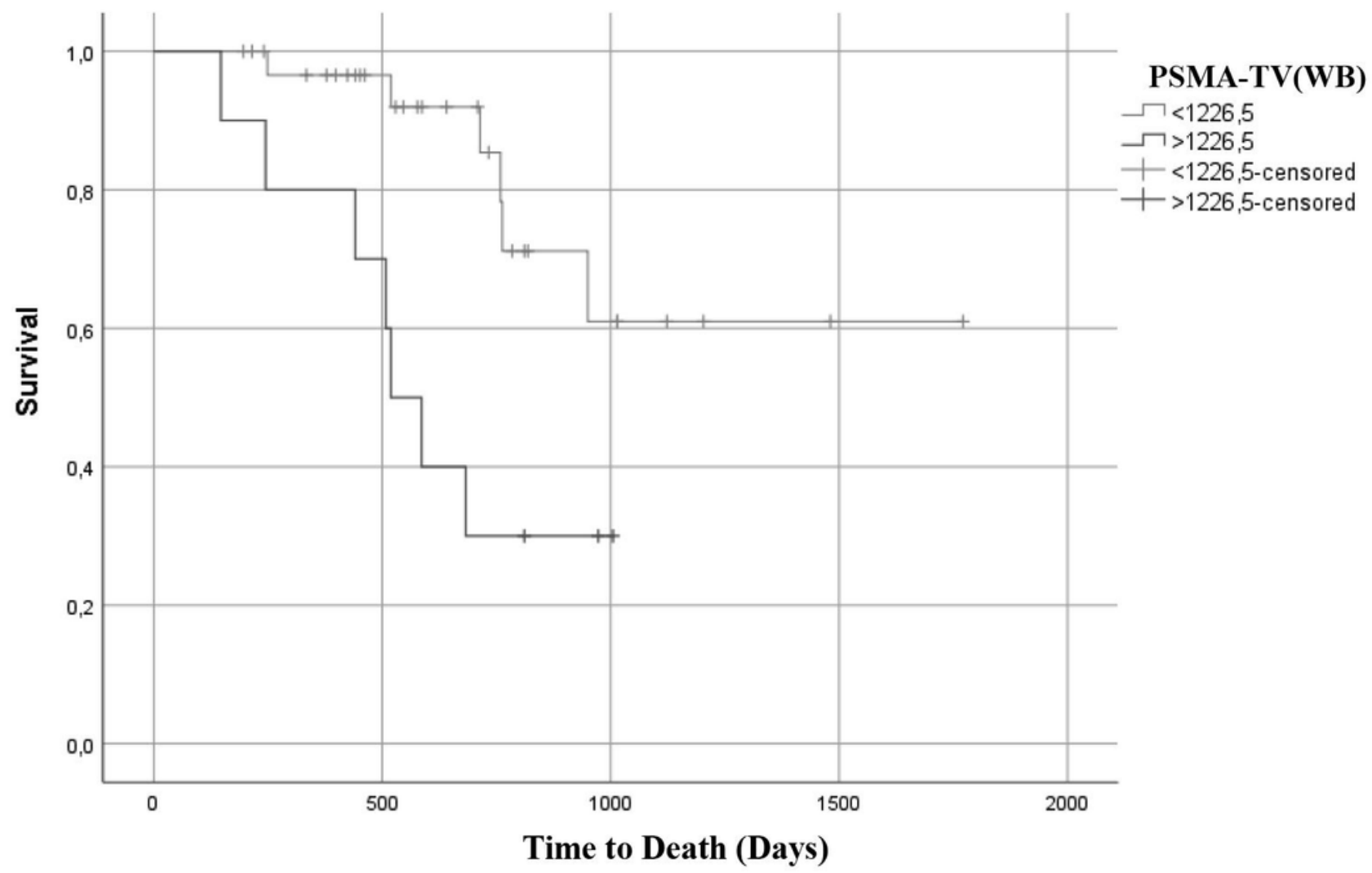

Figure 4

Legend not included with this version 